\title{
Radiation pressure on a moving body: beyond the Doppler effect
}

\author{
S. A. R. Horsley, ${ }^{1, *}$ M. Artoni, ${ }^{2,3}$ and G. C. La Rocca ${ }^{4}$ \\ ${ }^{1}$ School of Physics and Astronomy, University of St Andrews, North Haugh, St Andrews, KY16 9SS, UK \\ ${ }^{2}$ European Laboratory for Nonlinear Spectroscopy, Sesto Fiorentino 50019, Italy \\ ${ }^{3}$ Department of Physics and Chemistry of Materials CNR-IDASC Sensor Laboratory, Brescia University, Brescia 25133, \\ Italy \\ ${ }^{4}$ Scuola Normale Superiore and CNISM, Pisa 56126, Italy \\ *Corresponding author: sarh@st-andrews.ac.uk
}

Received July 31, 2012; revised September 10, 2012; accepted September 13, 2012; posted September 18, 2012 (Doc. ID 173632); published October 24, 2012

\begin{abstract}
The dependence of macroscopic radiation pressure on the velocity of the object being pushed is commonly attributed to the Doppler effect. This need not be the case, and here we highlight velocity-dependent radiation pressure terms that have their origins in the mixing of $s$ and $p$ polarizations brought about by the Lorentz transformation between the lab and the material rest frame, rather than in the corresponding transformation of frequency and wavevector. The theory we develop may be relevant to the nano-optomechanics of moving bodies. (C) 2012 Optical Society of America

OCIS codes: $\quad 350.5720,120.4880,160.3918$
\end{abstract}

\section{INTRODUCTION}

Radiation pressure arises from the momentum of light and depends upon the velocity of the body being pushed [1,2]. Take light incident onto the surface of a perfectly reflecting mirror that moves along the surface normal; in the mirror rest frame, the momentum and rate of arrival of the incident photons will appear either increased or decreased relative to the laboratory, depending on whether the mirror moves toward, or away from, the light source. This is a consequence of the Doppler effect, and implies that the velocity-dependent part of the radiation pressure acts to slow the mirror down. When attached to an oscillatory degree of freedom, this effect appears as a friction-like term in the equation of motion and is therefore termed radiation damping. For highly dispersive mirrors (e.g., a Bragg mirror), the reflection coefficient of the moving medium is also strongly dependent upon the Doppler shifted frequency, and this can turn damping into heating $[\underline{3}, \underline{4}]$.

The relative motion of a dielectric medium in general has a peculiar effect on the polarization of light as well as its propagation. The moving medium behaves as a magnetoelectric [5,6], which implies a change in the polarization composition $(s \& p)$ upon interacting with the medium. In spite of the fact that many features of reflection from, and transmission through, moving dielectrics have been considered before [-10], here we raise the question whether there exist optical forces, at least from a classical standpoint, arising from polarization mixing effects in a moving medium. (There has been some debate surrounding the role of the mixing of polarizations in the lateral Casimir force between moving plates (Casimir friction). On one side it has been argued that there should be a frictional force between the plates [11], but this calculation ignores the mixing of polarizations. Another calculation, which takes these into account [12], has claimed a zero value for the frictional force.) We find that there are situations where polarization mixing effects occur at the same order of $V / c$ as the Doppler effect, and cannot be neglected. Our theoretical framework, based on generalized scattering matrix methods, is readily amenable to single out these non-Doppler contributions to the radiation pressure experienced by a moving dielectric. We further examine a clear-cut configuration where the effect of radiation pressure is solely due to polarization mixing.

\section{TRANSFORMATION OF FIELD AMPLITUDES BETWEEN REFERENCE FRAMES}

The laboratory frame electromagnetic field, exerting pressure on a moving dielectric slab (see Fig. 1), can be written as

$$
\begin{gathered}
\mathbf{E}(\mathbf{x}, t)=\sum_{ \pm} \sum_{q=1,2} \hat{\mathbf{e}}_{q}^{( \pm)} \alpha_{q}^{( \pm)} e^{i\left(\mathbf{k}^{( \pm)} \cdot \mathbf{x}-\omega t\right)}, \\
\mathbf{B}(\mathbf{x}, t)=\frac{1}{c} \sum_{ \pm} \sum_{q=1,2}(-1)^{\bar{q}} \hat{\mathbf{e}}_{\bar{q}}^{( \pm)} \alpha_{q}^{( \pm)} e^{i\left(\mathbf{k}^{( \pm)} \cdot \mathbf{x}-\omega t\right),}
\end{gathered}
$$

where the surface has a normal parallel to $\hat{\mathbf{x}}, \mathbf{k}_{\|}=k_{y} \hat{\mathbf{y}}+k_{z} \hat{\mathbf{z}}$ and $\mathbf{k}^{( \pm)}= \pm\left|k_{x}\right| \hat{\mathbf{x}}+\mathbf{k}_{\|}$, with all the wavevector components real, and choosing polarization unit vectors $\hat{\mathbf{e}}_{1}^{( \pm)}=\hat{\mathbf{e}}_{1}=\hat{\mathbf{x}} \times \hat{\mathbf{k}}_{\|}$, $\hat{\mathbf{e}}_{2}^{( \pm)}=\hat{\mathbf{k}}^{( \pm)} \times \hat{\mathbf{e}}_{1}$, that are equivalent to $s(q=1)$ and $p(q=2)$ polarization. The barred index is defined as $\bar{q}=q+1(\bmod 2)$.

A Lorentz transformation of Eqs. (1) and (2) shows that for transverse motion $\mathbf{V}=V_{x} \hat{\mathbf{x}}$ the rest and laboratory frame field amplitudes are related by a constant of proportionality. For instance, inserting Eq. (1) and its primed counterpart into $E_{x}^{\prime}=E_{x}$, we have 


$$
\sum_{ \pm} \alpha_{2}^{\prime( \pm)}\left(\frac{c k_{\|}}{\omega^{( \pm)}}\right) e^{i\left(\mathbf{k}^{( \pm)} \cdot \mathbf{x}-\omega t\right)}=\sum_{ \pm} \alpha_{2}^{( \pm)}\left(\frac{c k_{\|}}{\omega}\right) e^{i\left(\mathbf{k}^{( \pm)} \cdot \mathbf{x}-\omega t\right)},
$$

where $\omega^{\prime( \pm)}=\gamma\left(\omega \mp V_{x}\left|k_{x}\right|\right)$ and $\gamma=\left(1-\mathbf{V}^{2} / c^{2}\right)^{-1 / 2}$. The equality (3) is satisfied if $\alpha_{2}^{( \pm)}=\left(\omega^{\prime( \pm)} / \omega\right) \alpha_{2}^{( \pm)}$, and examining the remaining components of the field, we find in general

$$
\alpha_{l}^{( \pm)}=\left(\omega^{( \pm)} / \omega\right) \alpha_{l}^{( \pm)}
$$

It is therefore evident that polarizations are not mixed by media moving in a direction parallel to their surface normal: the ratio of $s$ and $p$ polarizations will be maintained in transmission through and reflection from a transversely moving medium. This is the case for the radiation damping effect mentioned in the introduction, the physics of which is captured within Eq. (4).

Meanwhile for lateral motion $\left(\mathbf{V}=V_{y} \hat{\mathbf{y}}\right)$, we find that polarizations are mixed by the motion. Consider again the Lorentz transformation of the $x$ component of the electric field, Eq. (1), from the laboratory into the medium rest frame $E_{x}^{\prime}=\gamma\left(E_{x}+V_{y} B_{z}\right)$. Using Eqs. (1) and (2) this transformation gives the condition

$$
\begin{aligned}
\sum_{ \pm} \alpha_{2}^{( \pm)} \hat{e}_{2 x}^{( \pm)} e^{i\left(\mathbf{k}^{( \pm)} \cdot \mathbf{x}-\omega t\right)}= & \sum_{ \pm} \gamma\left[\alpha_{2}^{( \pm)}\left(\hat{e}_{2 x}^{( \pm)}-V_{y} \hat{e}_{1 z}^{( \pm)} / c\right)\right. \\
& \left.+\left(V_{y} / c\right) \alpha_{1}^{( \pm)} \hat{e}_{2 z}^{( \pm)}\right] e^{i\left(\mathbf{k}^{( \pm)} \cdot \mathbf{x}-\omega t\right)}
\end{aligned}
$$

where $\omega^{\prime}=\gamma\left(\omega-V_{y} k_{y}\right)$. After the components of the unit vectors have been written in terms of $\omega, \omega^{\prime}, \mathbf{k}$ and $\mathbf{k}^{\prime}$, Eq. (ㅁ) is equivalent to

$$
\alpha_{2}^{( \pm)}=\left(\frac{\omega^{\prime}}{\omega}\right) \frac{\alpha_{2}^{( \pm)} \mp\left(V_{y} \eta / c\right) \alpha_{1}^{( \pm)}}{\sqrt{1+V_{y}^{2} \eta^{2} / c^{2}}}
$$

with $\eta=\left|k_{x}\right| k_{z} /\left(k_{\|}^{2}-V_{y} \omega k_{y} / c^{2}\right)$. Examining the remaining components of the electric field reveals that the same relationship also holds for the $s$-polarization, but with $\eta \rightarrow-\eta$. This transformation of the field amplitudes can be summarized concisely in matrix form if we introduce the four-component vector (to avoid confusion we emphasize that despite having four components, $\alpha$ is not a relativistic four-vector), $\alpha^{T}=\left(\alpha_{1}^{(+)}, \alpha_{1}^{(-)}, \alpha_{2}^{(+)}, \alpha_{2}^{(-)}\right)$. Then the transformation can be written as

$$
\boldsymbol{\alpha}^{\prime}=\left(\frac{\omega^{\prime}}{\omega}\right) \mathbf{M} \cdot \boldsymbol{\alpha}
$$

with

$$
\mathbf{M}=\frac{1}{\sqrt{1+\frac{\eta^{2} V_{y}^{2}}{c^{2}}}}\left(\begin{array}{cc}
\mathbb{1}_{2} & \frac{V_{y} \eta}{c} \boldsymbol{\sigma}_{z} \\
-\frac{V_{y} \eta}{c} \boldsymbol{\sigma}_{z} & \mathbb{1}_{2}
\end{array}\right)
$$

and where $\boldsymbol{\sigma}_{z}$ is the usual Pauli matrix. We can now see that the situation is different for dielectric media in lateral motion where the electromagnetic field is modified both by the change of frequency and wavevector, and by the composition of the polarization [Eqs. (7) and (8)]. The unitary $4 \times 4$ matrix M describes how the two polarizations are coupled by the motion of the medium, and this specifically hinges on the of-diagonal $2 \times 2$ matrices $\pm V V_{y} \eta \boldsymbol{\sigma}_{z} / c$, which depend on the lateral velocity $V_{y}$ and the coefficient $\eta$. Such a polarization mixing effect vanishes for $k_{z}=0$ in which case the incidence plane contains the $y$ axis along which the slab moves and thus it remains a plane of mirror symmetry for the system assuring that $s$ and $p$ polarizations are independent. Conversely, when $k_{z} \neq 0$ and $k_{\|}^{2}=V_{y} \omega k_{y} / c^{2}$, we have $\eta \rightarrow \infty$, and the polarization is now completely converted from one type to another between reference frames.

\section{SCATTERING MATRIX}

We consider the radiation pressure for the case where we have right and left moving waves in the free-space regions, $x \leq$ $d / 2(\mathrm{~L})$, and $x>d / 2(\mathrm{R})$, as shown in Fig. 1 . In this case the slab is in lateral motion and the frequency, $\omega$ is conserved upon reflection and transmission.

In terms of the rest frame reflection and transmission coefficients of the slab $\left(r_{q}^{\prime}, t_{q}^{\prime}\right)$, for a single incident plane wave the rate of energy lost from the electromagnetic field is proportional to $1-\left|r_{q}^{\prime}\right|^{2}-\left|t_{q}^{\prime}\right|^{2}$, which owing to the translational symmetry in $y$ and $z$ is also proportional to the lateral force on the slab. Meanwhile the force on the slab in the $\hat{\mathbf{x}}$ direction is proportional to $1+\left|r_{q}^{\prime}\right|^{2}-\left|t_{q}^{\prime}\right|^{2}[\underline{13}]$. These results can be generalized and written in matrix notation as follows (for example, see $[\underline{14}, \underline{15}])$ :

$$
\left\langle\frac{d P_{M}^{\prime \mu}}{d t^{\prime}}\right\rangle=\frac{\epsilon_{0} A^{\prime} c^{2}\left|k_{x}^{\prime}\right|}{2 \omega^{\prime 2}} \boldsymbol{\alpha}_{\mathrm{IN}}^{\prime \dagger}\left(\begin{array}{c}
\left(\mathbb{1}_{4}-\mathbf{S}^{\prime \dagger} \mathbf{S}^{\prime}\right) \omega^{\prime} / c \\
\left(\mathbf{R}-\mathbf{S}^{\prime \dagger} \mathbf{R} \mathbf{S}^{\prime}\right)\left|k_{x}\right| \\
\left(\mathbb{1}_{4}-\mathbf{S}^{\prime \dagger} \mathbf{S}^{\prime}\right) k_{y} \\
\left(\mathbb{1}_{4}-\mathbf{S}^{\prime \dagger} \mathbf{S}^{\prime}\right) k_{z}
\end{array}\right) \boldsymbol{\alpha}_{\mathrm{IN}}^{\prime \dagger},
$$

where $\mathbf{R}=\boldsymbol{\sigma}_{z} \bigoplus \boldsymbol{\sigma}_{z}, A^{\prime}$ is the cross-sectional area of the beam of radiation as seen in the rest frame of the slab, and the triangular brackets indicate that we have dropped any rapidly oscillating interference terms from the force. Equation (9) can be verified using Eqs. (1) and (2), and calculating the

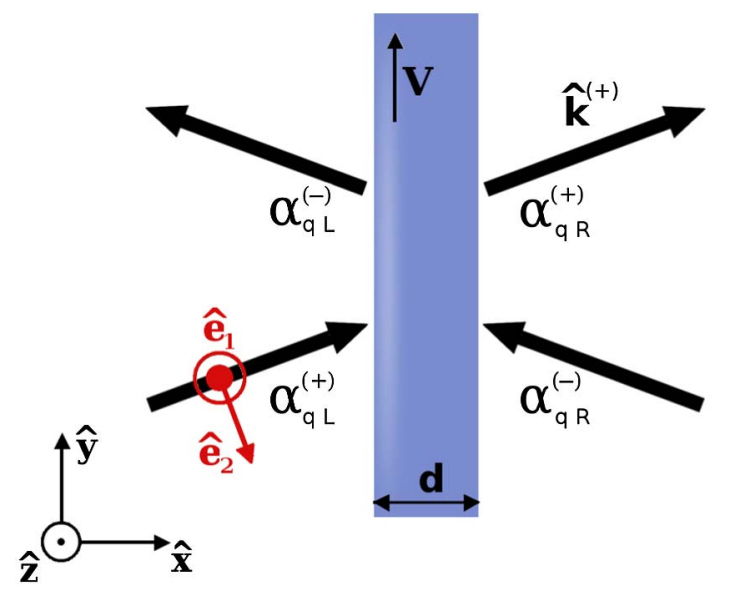

Fig. 1. (Color online) Scattering matrix $\tilde{\mathbf{S}}$ in Eq. (11) connects the laboratory frame input $\left(\alpha_{q L}^{(+)}, \alpha_{q R}^{(-)}\right)$and output $\left(\alpha_{q L}^{(-)}, \bar{\alpha}_{q R}^{(+)}\right)$amplitudes of fields scattering from a dielectric slab of thickness $d$ and area $A=$ $L_{y} L_{z}\left(L_{y}, L_{z} \gg d\right)$. The slab is centred at $x=0$, and moves laterally with velocity $\mathbf{V}$ and with surface normal parallel to $\hat{\mathbf{x}}$. The $s$ and $p$ polarization unit vectors, respectively, $\hat{\mathbf{e}}_{1}$ and $\hat{\mathbf{e}}_{2}$ and unit wavevector $\hat{\mathbf{k}}^{(+)}$are here displayed for the case of a plane wave propagating in the $(x-y)$ plane. More generally, the unit vectors $\hat{\mathbf{e}}_{1}$ and $\hat{\mathbf{e}}_{2}^{(+)}$lie in the $(\hat{\mathbf{z}}-\hat{\mathbf{y}})$ plane and in the incidence plane $\left(\hat{\mathbf{x}}-\hat{\mathbf{k}}_{\|}\right)$plane, respectively. Likewise for the incident wavevector that is written as $\mathbf{k}=k_{x} \hat{\mathbf{x}}+k_{\|} \hat{\mathbf{k}}_{\|}=$ $(\omega / c)[\cos (\theta) \hat{\mathbf{x}}+\sin (\theta)(\sin (\chi) \hat{\mathbf{y}}+\cos (\chi) \hat{\mathbf{z}})]$ where $\theta$ is referred to as the angle of incidence. 
energy momentum tensor of the electromagnetic field, and from this the rest frame four-force as in $[3,13]$. The incoming field amplitudes multiply the matrices inside the four-vector, and are written as $\boldsymbol{\alpha}_{\mathrm{IN}}^{\prime T}=\left(\alpha_{1 L}^{\prime(+)}, \alpha_{1 R}^{(-)}, \alpha_{2 L}^{(+)}, \alpha_{2 R}^{(-)}\right)$. The outgoing ones are $\boldsymbol{\alpha}_{\text {OUT }}^{\prime T}=\left(\alpha_{1 R}^{\prime(+)}, \alpha_{1 L}^{(-)}, \alpha_{2 R}^{(+)}, \alpha_{2 L}^{((-)}\right)$, and in the rest frame the relationship between the two is given by the scattering matrix, $\mathbf{S}^{\prime}\left(\omega^{\prime}, \mathbf{k}^{\prime( \pm)}\right): \boldsymbol{\alpha}_{\text {OUT }}^{\prime}=\mathbf{S}^{\prime} \cdot \boldsymbol{\alpha}_{\text {IN }}^{\prime}$. We assume a medium that does not mix $s$ and $p$ polarizations when at rest, so that the rest frame scattering matrix reduces to a direct sum, $\mathbf{S}^{\prime}=\mathbf{S}_{11}^{\prime} \oplus \mathbf{S}_{22}^{\prime}$, where

$$
\mathbf{S}_{q q}^{\prime}=\left(\begin{array}{cc}
t_{q}^{\prime} & r_{q}^{\prime} \\
r_{q}^{\prime} & t_{q}^{\prime}
\end{array}\right)
$$

Once $\mathbf{S}^{\prime}$ is known, this is equivalent to an exact solution of Maxwell's equations with the proper boundary conditions [16].

Performing a Lorentz transformation of Eq. (9) into the laboratory frame, where the slab is in motion, and applying the transformation of the field amplitudes in terms of Eq. (8), we find the following four-force in terms of laboratory frame field amplitudes,

$$
\left\langle\frac{d P_{M}^{\mu}}{d t}\right\rangle=\frac{\epsilon_{0} A c^{2}\left|k_{x}\right|}{2 \omega^{2}} \alpha_{\mathrm{IN}}^{\dagger}\left(\begin{array}{c}
\left(\mathbb{1}_{4}-\tilde{\mathbf{S}}^{\dagger} \tilde{\mathbf{S}}\right) \omega / c \\
\left(\mathbf{R}-\tilde{\mathbf{S}}^{\dagger} \mathbf{R} \mathbf{S}\right)\left|k_{x}\right| \\
\left(\mathbb{1}_{4}-\tilde{\mathbf{S}}^{\dagger} \tilde{\mathbf{S}}\right) k_{y} \\
\left(\mathbb{1}_{4}-\tilde{\mathbf{S}}^{\dagger} \tilde{\mathbf{S}}\right) k_{z}
\end{array}\right) \boldsymbol{\alpha}_{\mathrm{IN}}
$$

where $A=A^{\prime} / \gamma$. In Eq. (11), the effective laboratory frame scattering matrix is given by $\tilde{\mathbf{S}}=\mathbf{M}^{\dagger} \mathbf{S}^{\prime} \mathbf{M}$, which is a $4 \times 4$ matrix with all entries generally nonzero.

$$
\begin{aligned}
\tilde{\mathbf{S}} & =\mathbf{M}^{\dagger}\left(\mathbf{S}_{11}^{\prime} \oplus \mathbf{S}_{22}^{\prime}\right) \mathbf{M} \\
& =\frac{1}{1+\frac{\eta^{2} V_{y}^{2}}{c^{2}}}\left(\begin{array}{cc}
\mathbf{S}_{11}^{\prime}+\left(\frac{\eta V_{y}}{c}\right)^{2} \boldsymbol{\sigma}_{z} \mathbf{S}_{22}^{\prime} \boldsymbol{\sigma}_{z} & \frac{\eta V_{y}}{c}\left(\mathbf{S}_{11}^{\prime} \boldsymbol{\sigma}_{z}-\boldsymbol{\sigma}_{z} \mathbf{S}_{22}^{\prime}\right) \\
\frac{\eta V_{y}}{c}\left(\boldsymbol{\sigma}_{z} \mathbf{S}_{11}^{\prime}-\mathbf{S}_{22}^{\prime} \boldsymbol{\sigma}_{z}\right) & \mathbf{S}_{22}^{\prime}+\left(\frac{\eta V_{y}}{c}\right)^{2} \boldsymbol{\sigma}_{z} \mathbf{S}_{11}^{\prime} \boldsymbol{\sigma}_{z}
\end{array}\right)
\end{aligned}
$$

The conclusion we can draw from comparing Eq. (11) with Eq. (9) is that a laterally moving medium responds akin to a stationary medium with a peculiar dependence on frequency and wavevector (i.e., the $r_{q}^{\prime}$ and $t_{q}^{\prime}$ contain the rest frame frequency and wavevector), and reflection and transmission coefficients that are related to the rest frame reflection and transmission coefficients by a unitary transformation. At a frequency $\omega^{\prime}$ where the material has negligible loss, $\mathbf{S}^{\prime \prime} \mathbf{S}^{\prime}=\mathbb{1}_{4}$, all components of the four-force, besides $\left\langle d P_{M}^{1} / d t\right\rangle$, vanish, consistent with energy and momentum conservation. In terms of the rest frame scattering matrices, $\mathbf{S}_{q q}^{\prime}$, the effective laboratory frame scattering matrix takes the form (12), where the offdiagonal blocks linear in $\left(\eta V_{y} / c\right)$ are responsible for the polarization mixing effects.

\section{RADIATION PRESSURE EFFECTS DUE TO POLARIZATION MIXING}

The four-force in Eq. (11) along with Eq. (12) is an important result, as it embeds a new radiation pressure effect stemming from mixing of the $s$ and $p$ polarizations associated with moving frames. Two specific examples are now illustrated.
First, take the case of a plane polarized wave propagating in the direction $\hat{\mathbf{k}}^{(+)}$onto the left of a laterally moving slab, the two input polarization amplitudes being $\alpha_{1 L}^{(+)}$and $\alpha_{2 L}^{(+)}$. The resulting normalized (the tilde indicates that the force is in units of the incident average power $\epsilon_{0} c\left(\left|\alpha_{1 L}^{(+)}\right|^{2}+\left|\alpha_{2 L}^{(+)}\right|^{2}\right) A / 2$ divided by $c$ ) normal $(\hat{\mathbf{x}})$ force is

$$
\begin{aligned}
\left\langle\frac{d \tilde{P}_{M}^{1}}{d t}\right\rangle= & \frac{c^{2} k_{x}^{2}}{\omega^{2}\left(1+\eta^{2} V_{y}^{2} / c^{2}\right)}\left[1+\left(\left|r_{1}^{\prime}\right|^{2}-\left|t_{1}^{\prime}\right|^{2}\right)\left|\alpha_{1}+\frac{\eta V_{y}}{c} \alpha_{2}\right|^{2}\right. \\
& \left.+\left(\left|r_{2}^{\prime}\right|^{2}-\left|t_{2}^{\prime}\right|^{2}\right)\left|\alpha_{2}-\frac{\eta V_{y}}{c} \alpha_{1}\right|^{2}\right]
\end{aligned}
$$

which to leading order in $V_{y} / c$ is

$$
\begin{aligned}
\left\langle\frac{d \tilde{P}_{M}^{1}}{d t}\right\rangle & \simeq \frac{c^{2} k_{x}^{2}}{\omega^{2}}\left[1+\left(\left|r_{1}^{\prime}\right|^{2}-\left|t_{1}^{\prime}\right|^{2}\right)\left|\alpha_{1}\right|^{2}+\left(\left|r_{2}^{\prime}\right|^{2}-\left|t_{2}^{\prime}\right|^{2}\right)\left|\alpha_{2}\right|^{2}\right. \\
& \left.+\frac{\eta V_{y}}{c}\left(\left|r_{1}\right|^{2}-\left|t_{1}\right|^{2}-\left|r_{2}\right|^{2}+\left|t_{2}\right|^{2}\right)\left(\alpha_{1} \alpha_{2}^{\star}+\alpha_{1}^{\star} \alpha_{2}\right)\right],
\end{aligned}
$$

where we define $\alpha_{i}=\alpha_{i L}^{(+)} / \sqrt{\left|\alpha_{1 L}^{(+)}\right|^{2}+\left|\alpha_{2 L}^{(+)}\right|^{2}}$. The last expression is correct to first order in $V_{y} / c$ where $\eta \simeq\left|k_{x}\right| k_{z} / k_{\|}^{2}$, and the unprimed reflection and transmission coefficients depend on the laboratory frame frequency and wavevector rather than the rest frame values. We emphasize that we have not made any assumptions regarding the material properties of the slab, only that it can be characterized in the rest frame by a pair of reflection and transmission coefficients. Other terms linear in $V_{y} / c$ would appear upon expressing the primed reflection and transmission coefficients in terms of the laboratory frame quantities through the transformation of frequency and wavevector components. Such an expansion would bring out familiar Doppler terms [3,4] that are independent of the polarization-mixing terms proportional to $\left(\alpha_{1}^{*} \alpha_{2}+\right.$ c.c. $)$ shown on the right-hand side of Eq. (14). The normalized lateral $(\hat{\mathbf{y}})$ force, which vanishes for a lossless medium, takes a similar form,

$$
\begin{aligned}
\left\langle\frac{d \tilde{P}_{M}^{2}}{d t}\right\rangle \simeq & \frac{c^{2}\left|k_{x}\right| k_{y}}{\omega^{2}}\left[1-\left(\left|r_{1}^{\prime}\right|^{2}+\left|t_{1}^{\prime}\right|^{2}\right)\left|\alpha_{1}\right|^{2}-\left(\left|r_{2}^{\prime}\right|^{2}+\left|t_{2}^{\prime}\right|^{2}\right)\left|\alpha_{2}\right|^{2}\right. \\
& \left.+\frac{V_{y} \eta}{c}\left(\left|r_{2}\right|^{2}+\left|t_{2}\right|^{2}-\left|r_{1}\right|^{2}-\left|t_{1}\right|^{2}\right)\left(\alpha_{1} \alpha_{2}^{\star}+\alpha_{1}^{\star} \alpha_{2}\right)\right] .
\end{aligned}
$$

In general, polarization mixing contributions to the velocity dependent part of the force [Eqs. (14) and (15)] are comparable to the Doppler terms. (This holds for weakly dispersive media: whenever reflection and/or transmission have a strong dependence on frequency, such as in photonic bandgap media or close to an absorption line, the Doppler related effects can be amplified by many orders of magnitude [3,4].) Since they are proportional to ( $\alpha_{1}^{*} \alpha_{2}+$ c.c.), they vanish for unpolarized light, such as thermal radiation, where $\alpha_{1}$ and $\alpha_{2}$ do not have a definite mutual phase relation, and also for circularly polarized light $\left(\alpha_{1}=1 / \sqrt{2}, \alpha_{2}= \pm i / \sqrt{2}\right)$. Even when $\eta \neq 0$, the polarization mixing effects vanish to linear order in $V_{y} / c$ if in the lab frame the light is either purely $s$ or purely $p$ polarized, yet they survive and could be easily modulated or even flipped in sign by rotating the linear polarization when reflection and transmission for $s$ and $p$ polarizations are significantly different. In particular, for linearly polarized 
light with $\alpha_{1}=\sin \varphi$ and $\alpha_{2}=\cos \varphi$, corresponding to an electric field whose polarization vector makes an angle $\varphi$ with $\hat{\mathbf{e}}_{2}^{(+)}$, one has $\left(\alpha_{1}^{*} \alpha_{2}+\right.$ c.c. $)=\sin (2 \varphi)$.

Second, we consider a situation where radiation pressure arises only when both the mixing coefficient $\eta$ and the velocity do not vanish. Take two linearly polarized plane waves of equal intensity propagating in the direction $\hat{\mathbf{k}}^{(+)}$and $\hat{\mathbf{k}}^{(-)}$,
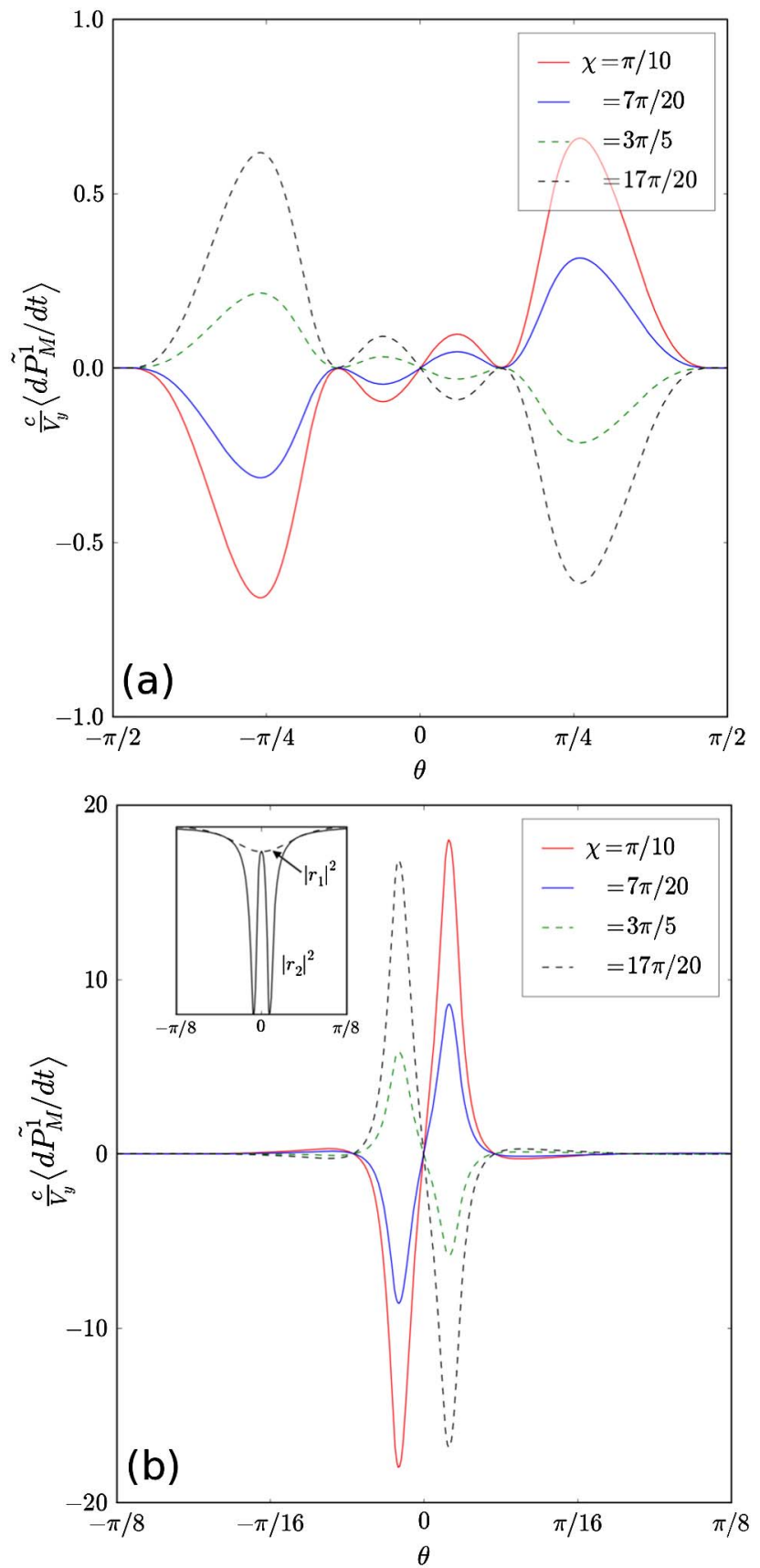

Fig. 2. (Color online) (a) Normal force exerted by a light beam of frequency $\omega$ on a laterally moving dielectric slab of thickness $d=$ $10 c / \omega$. The force, plotted as a function of the angle of incidence $\theta$, is computed from Eq. (16), where the optical response parameters $r_{i}$ and $t_{i}$ are evaluated using (a) $\epsilon=5.0+0.001 i$ and (b) $\epsilon=0.001+$ $0.001 i$ and $\mu=1$. For clarity we do not include the dispersion of the medium in this example, even though Eq. (16) can be applied to such a situation. The incident wavevector is defined as in Fig. 1. Inset shows the $s$ (dashed) and $p$ (solid) reflection coefficients for such a slab. This calculation was performed using the reflection and transmission coefficients for a slab of arbitrary thickness [16]. respectively onto the left and the right of a laterally moving slab. In much the same way as done above, the input field amplitudes may be chosen so that $\boldsymbol{\alpha}_{\mathrm{IN}}^{T}=\mathcal{E}\left(e^{i \zeta_{L}} \sin \left(\varphi_{L}\right)\right.$, $\left.e^{i \zeta_{R}} \sin \left(\varphi_{R}\right), e^{i \zeta_{L}} \cos \left(\varphi_{L}\right), e^{i \zeta_{R}} \cos \left(\varphi_{R}\right)\right)$, where $\Delta \zeta=\zeta_{L}-\zeta_{R}$ is the phase mismatch between the two input waves. Note that with our choice of polarizations the plane wave coming from the right and having $\varphi_{R}=\pi-\varphi_{L}$ is the mirror image of that coming from the left with respect to the $(z-y)$ plane of symmetry. Taking an average over $\Delta \zeta$ as appropriate for left and right incoming waves not having a definite mutual phase relation, the normal component of the force in this case can be obtained from Eq. (14) and the use of the mirror symmetry just described. In particular, for the specific choice $\varphi_{R}=\varphi_{L}=\varphi$ and retaining only terms linear in $V_{y} / c$, one has

$$
\left\langle\frac{d \tilde{P}_{M}^{1}}{d t}\right\rangle \simeq \frac{2 c\left|k_{x}\right|^{2} \eta V_{y}}{\omega^{2}} \sin (2 \varphi)\left(\left|r_{1}\right|^{2}-\left|t_{1}\right|^{2}-\left|r_{2}\right|^{2}+\left|t_{2}\right|^{2}\right)
$$

This force, which is present even when equal incident intensities are used (in both polarization channels) on both sides of the slab, is entirely due to the mixing of polarizations in the transformation described by Eq. (8) and does not seem to have been recognized before. In addition, the force (16), which does not contain Doppler contributions, is typically of the same order as the velocity-dependent radiation pressure stemming from the Doppler effect as mentioned in the introduction. Figure 2(a) shows the dependence of this force on the laboratory frame angle of incidence, $\theta$ when $\varphi=\pi / 4$. In this particular case we plot the effect for a nondispersive, absorbing thin dielectric slab with thickness $d$ comparable to the wavelength of the incident beam, although Eq. (16) can equally be applied to dispersive media.

For small (but not zero) $k_{\|}, \eta$ can become very large, and there is a large degree of polarization mixing. Although this is not relevant for ordinary materials, owing to the near degeneracy of $s$ and $p$ reflection and transmission coefficients at small angles of incidence, there are materials where the reflection and transmission coefficients remain significantly different close to normal incidence. This occurs, e.g., in epsilon nearzero metamaterials [17-19], and we plot in Fig. 2(b) the force on a slab with the same thickness but a refractive index three orders of magnitude smaller. Such an illustrative example shows more than an order of magnitude increase in the polarization mixing contribution to the velocity-dependent force.

\section{CONCLUSION}

We have derived a relativistic transformation law [Eq. (7)] to describe the change in polarization composition of an electromagnetic wave between inertial reference frames. This formula demonstrates that a laterally moving dielectric medium can mix polarizations, and that such effects are comparable in magnitude to those due to the Doppler shift. When applied to the theory of radiation pressure, this mixing of polarizations introduces new contributions to the force. In one example we have shown that a moving dielectric slab can experience a net force in the direction of the surface normal [Eq. (16)] even when radiation of the same intensity and direction is incident onto both sides of the slab.

The configuration leading to Eq. (16) is of special interest as it enables one to observe pure polarization mixing 
contributions to the velocity dependence of radiation pressure. This, in turn, can simply be tuned via the polarization angle; for instance, it can be turned on and off by setting $\varphi_{L}=$ $\pi / 4$ and switching $\varphi_{R}$ respectively to $\pi / 4$ and $3 \pi / 4$. Such an ability could be important in the emerging field of nanooptomechanics [20], where small optical forces can be used to manipulate the center of mass of mechanical oscillators of very light masses, or even atomic clouds [3]. In particular, it can allow one to control the dynamics of a two-dimensional oscillator in which the motion along one axis ( $\hat{\mathbf{y}}$ in our case) can be optomechanically coupled to that along the other one ( $\hat{\mathbf{x}}$ in our case).

\section{ACKNOWLEDGMENTS}

S. A. R. Horsley thanks the Engineering and Physical Sciences Research Council (EPSRC) for financial support. This work was also supported by the CRUI-British Council Programs "Atoms and Nanostructures" and "Metamaterials," and the IT09L244H5 Azione Integrata MIUR grant.

\section{REFERENCES}

1. V. B. Braginski and A. B. Makunin, "Ponderomotive effects of electromagnetic radiation," Sov. Phys. JETP 25, 653-655 (1967).

2. A. B. Matsko, E. A. Zubova, and S. P. Vyatchanin, "The value of the force of radiative friction," Opt. Commun. 131, 107-113 (1996).

3. S. A. R. Horsley, M. Artoni, and G. C. La Rocca,"Radiation damping in atomic photonic crystals," Phys. Rev. Lett.107, 043602 (2011).

4. K. Karrai, I. Favero, and C. Metzger, "Dopper optomechanics of a photonic crystal," Phys. Rev. Lett. 100, 240801 (2008).

5. L. D. Landau, E. M. Lifshitz, and L. P. Pitaevskii, The Electrodynamics of Continuous Media (Butterworth-Heinemann, 2004).
6. U. Leonhardt and T. G. Philbin, Geometry and Light: The Science of Invisibility (Dover, 2010).

7. C. Yeh, "Reflection and transmission of electromagnetic waves from a moving plasma medium,” J. Appl. Phys. 37, 3079-3082 (1966).

8. J. A. Kong and D. K. Chen, "Reflection and refraction of electromagnetic waves by a moving uniaxially anisotropic slab," J. Appl. Phys. 40, 2206-2212 (1969).

9. Y.-X. Huang, "Reflection and transmission of electromagnetic waves by a dielectric medium moving in an arbitrary direction," J. Appl. Phys. 76, 2575-2581 (1994).

10. M. Artoni, I. Carusotto, G. C. La Rocca, and F. Bassani, "Fresnel light drag in a coherently driven moving medium," Phys. Rev. Lett. 86, 2549-2552 (2001).

11. J. B. Pendry, "Shearing the vacuum-quantum friction," J. Phys. Condens. Matter 9, 10301-10320 (1997).

12. T. G. Philbin and U. Leonhardt, "No quantum friction between uniformly moving plates," New J. Phys. 11, 033035 (2009).

13. L. Novotny and B. Hecht, Principles of Nano-Optics (Cambridge University, 2008).

14. C. Genet, A. Lambrecht, and S. Reynaud, "Casimir force and the quantum theory of lossy optical cavities," Phys. Rev. A 67 , 043811 (2003).

15. B. A. Kemp, T. M. Grzegorczyk, and J. A. Kong, "Ab initio study of the radiation pressure on dielectric and magnetic media," Opt. Express 13, 9280-9291 (2005).

16. M. Born and E. Wolf, Principles of Optics (Cambridge University, 2009)

17. A. Alú, M. G. Silveirinha, A. Salandrino, and N. Engheta, "Epsilon-near-zero metamaterials and electromagnetic sources: tailoring the radiation phase pattern,” Phys. Rev. B 75, 155410 (2007)

18. L. V. Alekseyev, E. E. Narimanov, T. Tumkur, Yu. A. Barnakov, and M. A. Noginov, "Uniaxial epsilon-near-zero metamaterial for angular filtering and polarization control," Appl. Phys. Lett. 97, 131107 (2010).

19. J. Schilling, "Fundamental optical physics: the quest for zero refractive index," Nat. Photonics 5, 449-451 (2011).

20. F. Marquardt and S. M. Girvin, "Trend: optomechanics," Physics 2, 40 (2009). 